\title{
El Inca y sus curacas: poliginia real y construcción del poder
}

L'Inca et ses Curacas : polyginie royale et construction du pouvoir

The Inca and his curacas: royal polygyny and the construction of power

\section{Tom Zuidema}

\section{OpenEdition}

Journals

Edición electrónica

URL: http://journals.openedition.org/bifea/3284

DOI: 10.4000/bifea.3284

ISSN: 2076-5827

\section{Editor}

Institut Français d'Études Andines

\section{Edición impresa}

Fecha de publicación: 1 abril 2008

Paginación: 47-55

ISSN: 0303-7495

\section{Referencia electrónica}

Tom Zuidema, « El Inca y sus curacas: poliginia real y construcción del poder », Bulletin de l'Institut français d'études andines [En línea], 37 (1) | 2008, Publicado el 01 octubre 2008, consultado el 26 noviembre 2020. URL : http://journals.openedition.org/bifea/3284 ; DOI : https://doi.org/10.4000/ bifea.3284

Les contenus du Bulletin de l'Institut français d'études andines sont mis à disposition selon les termes de la licence Creative Commons Attribution - Pas d'Utilisation Commerciale - Pas de Modification 4.0 International. 


\title{
El Inca y sus curacas: poliginia real y construcción del poder
}

\author{
Tom Zuidema*
}

\section{Resumen}

Aunque tenemos pocos datos históricos en las crónicas sobre los antiguos curacas conquistados por los incas en los alrededores del Cuzco, tenemos datos importantes sobre las relaciones matrimoniales, políticas, calendáricas, rituales y mitológicas que los descendientes de éstos, reconocidos como «Incas de privilegio», mantuvieron con el Inca en el Cuzco. Un rol importante en estas relaciones fue dado a las esposas o hermanas de estos señores, las señoras Iñaca.

Palabras clave: Cuzco, matrimonio real, organización política, ritos, mitos

\section{L'Inca et ses Curacas : polyginie royale et construction du pouvoir}

\section{Résumé}

Alors que les chroniques ne nous fournissent que peu d'information historique sur les anciens Curacas des alentours du Cuzco conquis par les Incas, nous possédons en revanche de nombreux éléments importants sur les relations matrimoniales, calendaires, politiques, rituelles et mythologiques que les descendants de ces Curacas, reconnus comme des «Incas par privilège», ont entretenues au Cuzco avec l'Inca. Au coeur de ces relations, les «Dames Iñaca», épouses ou soeurs des ces Seigneurs, jouaient un rôle essentiel.

Mots clés : Cuzco, mariage royal, organisation politique, mythes, rites 


\title{
The Inca and his curacas: royal polygyny and the construction of power
}

\begin{abstract}
Although we have little historic data from the chronicles on the ancient curacas conquered by the Incas in the area around the city of Cuzco, we do have important data on the matrimonial, political, calendrical, ritual and mythical relations that the descendants of the curacas - known as the «lncasby-privilege»_ - had with the Inca in the city of the Cuzco. The wives or sisters of these authorities, the señoras Iñaca, played an important role in these relations.
\end{abstract}

Key words: Cuzco, royal marriage, political organization, myths, ritual

Si comparamos las crónicas sobre Cuzco, capital inca, y sus alrededores con aquellas de Tenochtitlán, capital azteca, con sus alrededores, notamos una gran diferencia. En México, cronistas indígenas de ciudades cercanas a la capital, como por ejemplo,Tezozómoc, Chimalpáin e Ixtlilxóchitl, se interesaron por las historias heroicas de sus propios pueblos y así podemos situar nuestra información dinástica, política, calendárica, ritual, etc. sobre Tenochtitlán en un marco comparativo, crítico e histórico. Y esto, sin referirme siquiera, en un marco más amplio, a las grandes crónicas y códices sobre Tlaxcala, los tarascos, mixtecas, zapotecas, mayas, etc... En el Perú, casi toda la atención de los cronistas se concentró sobre la ciudad del Cuzco, con la excepción de la provincia de Huarochirí (Taylor, 1999). Hay alguna información legendaria sobre las capitales de pueblos cercanos al Cuzco, sujetos por los incas en un pasado no más lejano que los cercanos a Tenochtitlán en México, y hasta conocemos algunos mitos o leyendas sobre sus antiguos curacas, pero sus reinos no atrajeron a los cronistas. Nos falta también una crónica sobre los antiguos reinos colla, cerca del lago Titicaca. El rico manuscrito de Huarochirí no dice casi nada de sus gobernantes anteriores, y lo poco que sabemos sobre el antiguo reino de Chimor concierne más bien la mitología del reino anterior de Ñampallec (Cabello Valboa, 1951; Rowe, 1948; Zuidema, 1990).

Quizás el interés por la historia prehispánica de los intelectuales españoles e indígenas en los Andes no fuera mucho menor que el de Mesoamérica, si consideramos que el tipo de información que podían recoger fue muy distinto. Betanzos, nuestro primer cronista sobre la cultura inca, es bien explícito en declarar que el patrón dinástico — hacia 1550 - que el virrey Don Antonio de Mendoza le obligó a reconstruir y a reportar sobre el Cuzco, fue de muy poca relevancia para los mismos andinos para explicar y defender su sistema político y la continuidad de éste (Betanzos, 1987 [1551]: 7). Al revés, fue el creciente interés español, culminando con la intervención del virrey Don Francisco de Toledo cuando obligó a su cronista Pedro Sarmiento de Gamboa [1572] a que interpretara la memoria inca en forma de herencia dinástica de padre a hijo, lo que destruyó efectivamente la concepción específica inca de la memoria del pasado y de su concepto de continuidad en el gobierno (Sarmiento de Gamboa, 1943 [1572]; Zuidema, 2005a). No obstante, rasgos de esta memoria inca sobrevivieron en las crónicas. Y como estos rasgos se conforman mejor con el sistema real de política inca, aportan más datos explicativos y ofrecen más posibilidades de análisis suplementarios. Aquí me concentraré en un aspecto crucial del significado para el estado de la poliginia del Rey en el sistema político, que resultó de esto 
y en su continuidad en el tiempo. Tal como resulta en otras partes de los Andes, nuestra información es más bien de carácter calendárico, ritual y legendario o mitológico, pues así fue como los incas se expresaron - y lo observó claramente Betanzos-. Es la información más empírica que ellos nos dieron. Siguiendo este camino de investigación, el contenido de ciertos documentos históricos también resulta ser más prometedor.

La descripción más explícita del sistema poligínico del Rey la debemos al cronista Pedro Pizarro quien trabó amistad con el último rey inca, Atahuallpa, en su prisión en Cajamarca. Es el único testimonio directo sobre un Inca reinante en su corte y de su modo de gobernar. Un detalle del mismo sistema de gobierno lo observaron los españoles en la tarde anterior a la derrota del imperio. Dice Pedro Pizarro que aparte de la Coya, la Reina, el Inca tenía a otras 40 mujeres, cada una acompañada por un servicio de 100 vírgenes provenientes de todas las partes del país. Cada esposa en turno servía al Rey por un periodo de 8 a 10 días, hasta que el turno volvía a la primera (Pizarro, 1978 [1572]: 78). Cuando los españoles se entrevistaron con Atahuallpa en el palacio de los baños, las únicas personas que estuvieron con él adentro, sirviéndole, fueron aquellas señoras mencionadas por Pedro Pizarro. Después de Atahuallpa, esta organización se perdió pero no su memoria pues un documento sobre la participación de un descendiente de Atahuallpa en una fiesta quiteña del año 1630, todavía se refiere a dicha organización en detalle; menciona cómo al lado del Rey y de sus ocho capitanes salieron la reina de Cochasquí y cuarenta «mugeres» como damas de su corte (Herrera, 1916: 88). Otros elementos apuntados por cronistas del Cuzco nos permiten reconstruirla de forma más completa.

Cuando Betanzos [1551], nuestro primer cronista, describe las tareas de las muchachas de 5 a 15 años, especifica que estaban bajo el cuidado de ciertas señoras que se turnaban cada 10 días. Él define a éstas Cuzco iñaca cuna, es decir señoras Iñaca del Cuzco, como «ciertas matronas romanas», las que inspeccionaban a las mujeres que entraban en la ciudad (Betanzos, 1987 [1551], I. 1: cap. 21). Reconocemos en ellas las esposas secundarias mencionadas por Pedro Pizarro.

Veinte años más tarde, Sarmiento [1572] hace coincidir su descripción de las señoras Iñaca con un relato, probablemente inspirado por Betanzos, de la boda de Pachacuti Inca, el gran Rey organizador del Cuzco, con una señora de las afueras del Cuzco en el mismo valle (Sarmiento de Gamboa, 1943 [1572]: cap. 47). Ella había tomado su nombre de la mítica ancestra preincaica de su pueblo, Mama Anahuarque. Según Betanzos, recibió de su marido el servicio de 100 acllas, vírgenes escogidas, y del sacerdote del Sol 50 acllas más. En la versión de Sarmiento, quizás más cercana a la verdad, Pachacuti tuvo, aparte de sus hijos legítimos con Mama Anahuarque, a100 hijos y a 50 hijas «bastardos», que «por ser muchos, llamó Hatun aillo (gran linaje) y por otro nombre... Iñaca panaca aillo», o sea, «los varios linajes descendientes del Rey en mujeres secundarias Iñaca». Los dos nombres del ayllu o panaca pertenecían al mismo grupo de gente y así lo entendieron también cronistas posteriores. Reuniendo la información de Pizarro sobre las 40 otras esposas, cada una con un servicio de 8 ó 10 días, la de Betanzos sobre las señoras Iñaca, también con servicio por 10 días y la de Sarmiento sobre las mujeres secundarias del Rey, toma cuerpo la identificación entre los tres grupos mencionados. El servicio de 100 acllas que solo Mama Anahuarque recibía, según Betanzos, o cada una de las esposas, según Pizarro, en palabras de Sarmiento se transforma en 100 hijos y 50 hijas bastardas del Rey. 
No encontramos en Betanzos ni en Sarmiento el número de 40 como total de las señoras Iñaca, pero el primero sí aludió a ello en parte cuando en un comentario anterior, dijo que Pachacuti había recibido 20 esposas al asumir el trono. Y habló entonces de 10 jefes no-Incas de más alto rango y de 10 de menor rango. Reconocemos también el número 40 en las dos descripciones más tardías, bien paralelas, de Felipe Guaman Poma de Ayala y de Martín de Murúa (Guaman Poma, 1987 [1615]: 757-760; Murúa, 1964 [1613], I. 2: 62-63). Ellos discuten la organización de tipo decimal de la región alrededor del Cuzco incluyendo los pueblos cuyos curacas habían recibido el privilegio de llevar el título de Inca y de no pagar tributo. La región, definida como provincia o huamani de 40000 familias, fue subdivida en entidades de 20 000, 10 000, 5 000, 1 000, 500 y menores números de familias. Las señoras Iñaca fueron esposas y/o hermanas de los 40 curacas de huaranca, de mil familias cada una, y solo éstos fueron Incas de privilegio, un rango limítrofe entre Inca y no-Inca. Mientras que las señoras de más alto rango consideradas como «hijas» del Rey eran casadas en el Cuzco de mano del mismo Inca, las señoras Iñaca solo lo fueron en presencia del Rey pero no de su mano. Las Iñacas ocuparon un rango intermediario entre «hijas» y «nohijas», como esposas secundarias en quienes el Inca procreó descendencias «bastardas». En efecto, Betanzos (1987 [1551], cap. 16: 78) definió a los miembros de diferentes linajes, que podemos concluir pertenecieron a Iñaca panaca (grupo de descendientes de una pana «hermana de un hombre»), no como «hijos del Inca» sino como huaccha concha, «sobrinos pobres» (huaccha «pobre, huérfano»; concha «hijo/a de la hermana pana de un hombre») (Zuidema, 2005a).

La última información proviene de Garcilaso de la Vega, el Inca [1609] y es muy reveladora sobre el verdadero rol en la corte de las señoras Iñaca, aunque parece desconocer el término y quizás también la antigua posición legal de aquellas en la sociedad cuzqueña (Garcilaso de la Vega, 1991 [1609], I. 3: cap. 22). Después de explicar las semanas incaicas, dice que unos representantes de los diferentes pueblos de Incas de privilegio ejercían distintos oficios en los templos y en la corte del Cuzco. En la mesa del mismo Inca solamente servían «sus mujeres concubinas» y ellas defendían los intereses de sus pueblos con el mismo Rey. «Se remudaban de tantos a tantos días, semanas o meses» y mientras Garcilaso aquí no adhiere al patrón estricto de semanas, sí implica que tuvieron una relevancia aún mayor en el calendario para todo el sistema político y jerárquico inca del Cuzco.

La información reunida de las crónicas me permite ahora anclar una reconstrucción bastante completa de una organización de 40 señoras Iñaca en el sistema de los Ceques; es decir, el registro de 328 huacas del valle del Cuzco por medio de 41 direcciones, los ceques, y del culto a los 41 ceques por igual número de grupos sociales. Vale por eso integrar al sistema también la región aledaña, bajo la autoridad de los Incas de privilegio. Asumo que cada señora Iñaca llegaba a la corte siguiendo su propio ceque. Aparte de un único ceque llamado Capac — calificado de «real» probablemente por estar relacionado con la Reina10 ceques estuvieron a cargo de los 10 grupos de mayor rango, mencionados por Betanzos. Cronistas posteriores nombraron estos grupos como las 10 panacas; otros 10 ceques estuvieron a cargo de los 10 grupos de menor rango, que los demás cronistas conocieron como los «10 ayllus» del valle del Cuzco. El gran rito de la Situa, en septiembre, sugiere la inclusión de un rol de los 20 ceques restantes. Cuatrocientos guerreros iban a echar los males y las enfermedades a los confines del territorio de los Incas de privilegio, dividido éste a la vez en cuatro partes o suyus así como en cuatro bandas de jerarquía descendiente 
alrededor del Cuzco. Los hombres hacían el recorrido en relevo: primero 100 hombres de las 10 panacas, entonces 100 de los 10 ayllus, y después dos veces 100 hombres de los Incas de privilegio que atravesaban sus propios territorios (Molina, 1989 [1574]: 73-75). Estos últimos grupos bien podían haber entregado al Rey en el Cuzco a las 20 señoras Iñaca que todavía faltaban de las 40 totales.

Nuestras fuentes nos sugieren varios nombres propios de estas señoras y quiero terminar mi análisis muy general del sistema mencionando a algunas de ellas. Pero antes de seguir, necesito recordar dos factores a tomar en cuenta. No todas las 40 señoras podían corresponder al rango que Guaman Poma y Murúa habían adjudicado a las Iñaca: es decir, no solo hermanas o esposas de curacas de Incas de privilegio sino también y a la vez, de curacas de huarancas (1 000 familias). Vale por eso mencionar el primer significado del término iñaca por el cual ellas se distinguían: designa la fina tela, llamada tanto ñañaca como iñaca, que en forma plegada ellas llevaban sobre la cabeza. Guaman Poma enseña que las señoras Iñaca constituyeron el último rango inferior que tenía permitido llevar la ñañaca pues las señoras de rangos más altos también la usaban. Podemos concluir que los 41 ceques correspondían a 41 señoras de distintos rangos de Incas y de Incas de privilegio, incluyendo a la Reina, a las señoras de panacas, de ayllus y de Incas de privilegio, pero no exclusivamente a las señoras Iñaca.

Un interés inicial que me instigó a estudiar a las señoras Iñaca radica en el calendario estatal. Como ya nos indicaron los primeros cronistas, el sistema de ceques funcionó como una organización tanto espacial como temporal, o sea como calendario (Zuidema, en prensa). Una información abundante aunque dispersa nos permite reconstruir la secuencia diaria de las 328 huacas por medio de su distribución sobre los 41 ceques. El promedio de huacas por ceque era de 8 , el número que ya mencionó Pizarro, pero el número real de huacas por cada ceque variaba mucho, probablemente por influencia de la posición de la señora asociada al ceque en el sistema jerárquico. El sistema de ceques como calendario indica cuando una señora particular estaba presente en la corte y qué rol entonces iba a cumplir allí en el marco de los ritos del calendario anual.

Si ahora puedo identificar a varias señoras Iñaca y a los pueblos que representaban, observamos un rasgo bastante peculiar de la cultura inca andina. Estas señoras no fueron recordadas tanto como personas de carne y hueso sino por las huacas, cerros, mitos y ritos que se asociaban a ellas. Obtenemos también más información pertinente por medio de los mitos. En la memoria inca del pasado, a las antiguas representantes de las panacas se les asignó el rango de Coyas, es decir Reinas, y conocemos algunos de sus pueblos de origen no-inca por sus huacas como por ejemplo cerros. Y podemos identificar ceques dirigidos hacia aquellos pueblos. En las crónicas y documentos, tenemos acceso a varias listas de pueblos de Incas de privilegio, aunque todavía es difícil precisar cuáles pueblos, y en qué orden, participaban en el orden calendárico de las señoras Iñaca (Zuidema \& Poole, 1982). Al cumplir con esta tarea tenemos que aceptar también cierta inventiva de parte de las entidades participantes, tal como lo vemos en pueblos actuales. Por ejemplo, un pueblo, o pueblos como dice Garcilaso, y su señora Iñaca podría representar un ceque en una oportunidad y otro ceque en otra. Sospecho que podemos conseguir todavía documentación etnohistórica en los pueblos descendientes de Incas de privilegio y de sus señoras Iñaca, no solo con el propósito de conocer su pasado histórico, sino para entender en qué consistían su participación y su contribución a la organización estatal del Cuzco y de su región, en tiempos incaicos y hasta en los primeros años de la Colonia. 
Quiero terminar presentando tres casos de señoras Iñaca y de sus lugares en el calendario político-ritual. En mi representación del calendario de los ceques doy a cada día el espacio de un grado en un compás de 365 grados; hay 12 meses de duración desigual y un décimotercer mes compuesto por los días que no se contaban cuando no había cultivo en las tierras del estado (Zuidema, 1990; en prensa). Los tres casos están bien documentados por una razón que comprendí solo posteriormente. Sarmiento (1943 [1572]: cap. 52) nos revela que la gente común tenía que trabajar en las tierras del Inca todo el año excepto durante tres meses cuando podían dedicarse a sus propias chacras. El primer mes era para arar y sembrar, el segundo para cosechar, y el tercero, según el cronista, «en el verano, para sus fiestas y para que hilasen y tejiesen para sí». Fueron éstos los meses Incas correspondientes a septiembre y abril, y el mes correspondiente a mayo. Según la descripción del sistema de los ceques, los trabajadores fueron representados por aquellos ceques o grupos de ceques que no tenían conexión con ninguna panaca (véase la figura 1: las panacas se indican aquí con los números 1-10 y los meses para la gente común por los nombres de las tres señoras).

Figura 1 - La representación calendárica del sistema de los ceques con la participación de algunas señoras míticas del rango Iñaca

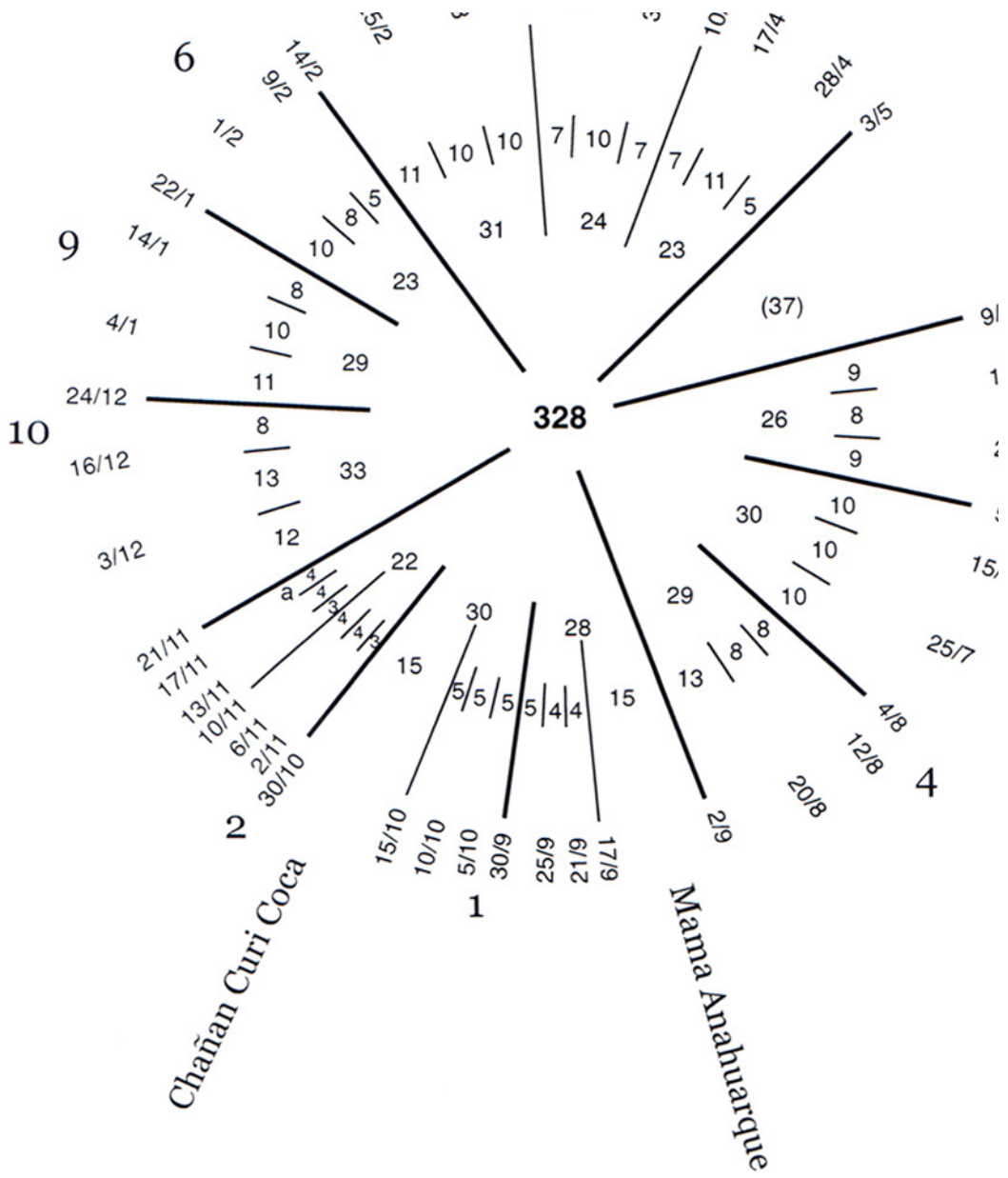


El primer ejemplo es de Mama Anahuarque, la Coya del Inca Pachacuti, el cual procreó a muchos hijos secundarios en señoras Iñaca de todas partes de su nuevo reino. Aunque de origen no-inca, como esposa principal iba a ser reconocida como reina. Fue nombrada por el cerro Anahuarque y por el ceque dirigido a este cerro como huaca de su pueblo nativo, Choco, cerca del Cuzco (Sarmiento, 1943: caps. 34, 47). Sus habitantes reclamaron derechos autóctonos sobre sus tierras pues sus antepasados preincaicos habían sobrevivido sobre su cerro cuando éste fue el único que subió con las aguas del Diluvio (Molina, 1989: 104-106). Mama Anahuarque — cerro y coya - eran venerados en «la fiesta de la Reina», Coya raymi, por septiembre, dedicada a la siembra, a la fertilidad de la tierra, en honor, precisan las crónicas, a la Luna, a la Coya y a todas las mujeres, y celebrada por Incas e Incas de privilegio. La manera más explícita de expresar esta dedicación consistía en las grandes carreras de los muchachos por iniciar, desde el cerro hasta el Cuzco, cuando las muchachas que eran sus acompañantes ya se les habían anticipado para recibirles abajo con chicha (cerveza de maíz) (Zuidema \& Urton, 1976). En ciertas fiestas actuales por septiembre, fuera del Cuzco, los hombres jóvenes todavía se visten con trajes de mujer, lo que subraya el carácter femenino de la celebración (Arguedas, 1956). Es bien probable que a principios de Coya raymi una mujer de Choco, con el mismo nombre Anahuarque del cerro y de su antepasada mítica y legendaria, representara a su pueblo en la corte del Inca. Un reflejo de la relación se detecta todavía en documentos del siglo XVII que Horacio Villanueva y Jeanette Sherbondy publicaron sobre el calendario de riego en el valle del Cuzco, donde cada familia tenía derecho a cierta cantidad de días y a ciertas fechas para regar (Villanueva \& Sherbondy, 1979). Describen cómo en este contexto los habitantes de Choco seguían manteniendo sus antiguos y distintivos privilegios de origen preincaico.

El segundo ejemplo trata de Mama Huaco, la cuarta hermana de Manco Cápac, el mítico fundador del Cuzco (Pachacuti Yamqui Salcamaygua, 1993: 183-187; Sarmiento de Gamboa, 1943: cap. 13; Zuidema, 2005b). Como dos de los tres hermanos fueron reconocidos en el sistema de ceques como antepasados de ayllus «comunes» podemos concluir que ella también representara un rango inferior similar. Conquistó la ciudad en dos ocasiones: en la primera sembró el primer maíz y en la segunda, un año más tarde, lo cosechó. Más singular todavía iconocemos exactamente su camino de conquista! Siguió un ceque (el que representa el periodo de 10/4-17/4), que resulta ser el único documentado fuera de la relación de los ceques. El gran rito estatal por abril en tiempo de la cosecha, una procesión en que participaba el Inca y la nobleza, también seguía esta misma ruta; en esta ocasión se recogía la cosecha de la muy conocida chacra, llamada Sausero, que fue dedicada a ella y a su momia (Segovia, 1968 [1552]: 81-82). La chacra fue propiedad de los descendientes del último Inca impuesto por los españoles, Paullu Inca, quien murió en 1549. Sospecho, sin embargo, que el pueblo de Incas de privilegio y del mismo nombre de Paullu, cerca de Pisac, también tuvo algo que ver con la chacra Saucero (Cobo, 1956: 214-215; Molina, 1989: 118-119). Mama Huaco fue adorada en forma de una olla, vestida de mujer, que contenía la semilla de maíz del año por venir (Cobo, 1956: 215). Queda la pregunta del significado de su momia pues no sabemos a cuál grupo originalmente perteneció y así cuáles ayllus o pueblos la adoraron. Sospecho que fueron aquellos que vivían en el valle más abajo del ceque que definió la ruta mítica por la cual Mama Huaco entró al Cuzco.

El último ejemplo es de la señora Chañan Cori Coca. En un primer contexto mítico, ella había sido una mujer valiente que en la defensa del Cuzco por Pachacuti contra los Chancas había vencido a los enemigos atacándolos hacia el Sur (Sarmiento, 1943: cap. 27). Como tal, tenemos de ella un retrato colonial, conservado en el museo arqueológico del Cuzco. Su sepultura fue la huaca de un ceque (aquel indicado por su nombre) en la misma 
dirección al sur (Cobo, 1956: 184). En el segundo contexto mítico, bien investigado por Gary Urton en base a un documento del siglo XVI, ella habría sido la ancestra — digamos la señora Iñaca - del ayllu más importante de Pacari tambo, el mítico lugar de origen de los Incas (Urton, 1990: 46-62). Está bien documentado que los Incas de privilegio de los alrededores de Pacaritambo, los masca, fueron representados también por un ayllu suyo en el valle del Cuzco. La relación entre Pacaritambo y Cuzco no es solamente, y ni siquiera principalmente, mítica. Cada año, hoy día, como herencia de su antigua obligación de mita, los hombres de Pacaritambo todavía renuevan el camino entre su pueblo y el Cuzco. En los años cincuenta yo he podido presenciar cómo la gente de dicho pueblo, cuando emigraba a la ciudad, se quedaba a vivir en su entrada, por la zona del pueblo de Choco. Los emigrantes habían formado allí un ayllu con el nombre de su pueblo. Esta costumbre parece ser más general entre gente de los pueblos de los alrededores del Cuzco. Lo que nos falta por conocer en este ejemplo de Chañan Cori Coca es la conexión calendárica entre Pacaritambo y Cuzco. El calendario inca sugiere una semana al principio de octubre pero hay que buscar argumentos en su apoyo.

Podemos documentar en forma similar los casos de otras señoras Iñaca, de sus pueblos y sus ceques, y quizá de sus obligaciones al Cuzco; obligaciones que Molina (1989: 74-75) define como las de mitimaes. Pero, para ello, tendría que incluir también otro tipo de información, especialmente sobre las panacas y su rol calendárico, un aspecto al cual no puedo dedicar más espacio en este artículo. Espero que, con las posibilidades que el ejemplo de Pacaritambo nos ofreció, encontremos ejemplos similares de otros antiguos pueblos de Incas de privilegio. Serían de valor no solamente para conocer a estos pueblos sino también para establecer una base más amplia del estudio del Cuzco incaico en su contexto regional inca y colonial.

\section{Referencias citadas}

ARGUEDAS, J. M., 1956 - Puquio, una cultura en proceso de cambio. Revista del Museo Nacional, 25: 184-232; Lima.

BETANZOS, J. de, 1987 [1551] - Suma y Narración de los Incas (Martín Rubio, ed.), 317 p.; Madrid: Atlas.

CABELlO VAlBOA, M., 1951 - Miscelánea Antártica. Una Historia del Perú Antiguo, 561 p.; Lima: Facultad de Letras de la Universidad Nacional Mayor de San Marcos, Instituto de Etnología. Con prólogo, notas e índices a cargo del Instituto de Etnología. Seminario de historia del Peru-Incas.

COBO, B., 1956 - Historia del Nuevo Mundo; Madrid: Atlas. Biblioteca de Autores Españoles, vol. 92.

GARCILASO DE LA VEGA, I., 1991 [1609] - Comentarios Reales de los Incas; Lima, México, Madrid: Fondo de Cultura Económica. Edición, prólogo, índice analítico y glosario de Carlos Araníbar.

GUAMAN POMA DE AYALA, F., 1987 [1615] - Nueva Crónica y Buen Gobierno (J. V. Murra, R. Adorno \& J. L. Urioste, eds.); Madrid. 
HERRERA, P., 1916 - Apunte Cronológico de las Obras y Trabajos del Cabildo y Municipalidad de Quito desde 1534 hasta 1714; Quito. Primera parte.

MOLINA, C., 1989 [1574] - Fábulas y Mitos de los Incas (H. Urbano, ed.): 1-136; Madrid. Historia 16.

MURÚA, M. de, 1964 [1613] - Historia General del Perú (M. Ballesteros Gaibrois, ed.); Madrid: Instituto Gonzalo Fernández de Oviedo.

PACHACUTI YAMQUI SALCAMAYGUA, J. de S. C., 1993 - Relación de Antigüedades Deste Reyno del Pirú (Pierre Duviols \& César Itier, eds.), 276 p.; Cusco: Institut Français d'Études Andines, Centro de Estudios Regionales Andinos «Bartolomé de Las Casas»).

PIZARRO, P., 1978 [1572] - Relación del descubrimiento y conquista de los Reinos del Perú (Lohmann Villena, ed.), 277 p.; Lima: Fondo Editorial de la Pontificia Universidad Católica del Perú.

ROWE, J. H., 1948 - The kingdom of Chimor. Acta americana, 6(1-2): 26-59; Uppsala.

SARMIENTO DE GAMBOA, P., 1943 [1552] - Historia de los Incas (Angel Rosenblatt, ed.), 302 p.; Buenos Aires: Émécé editores.

SEGOVIA, B. de, 1968 - Relación de muchas cosas acaescidas en el Peru (Aquí atribuida a Cristóbal de Molina, el Almagrista) (Francisco Esteve Barba, ed.): 56-96; Madrid: Atlas. Biblioteca de Autores Españoles, Vol 209. Crónicas Peruanas de Interés Indígena.

TAYLOR, G., 1999 - Ritos y Tradiciones de Huarochirí (G. Taylor, ed.), 502 p.; Lima: Instituto Francés de Estudios Andinos, Banco Central de Reserva del Perú, Universidad Particular Ricardo Palma. Segunda edición revisada.

URTON, G., 1990 - The History of a Myth. Pacariqtambo and the Origin of the Inkas; 172 p.; Austin: University of Texas Press.

VILLANUeVA, H. \& SHERBONDY, J. E., 1979 - Cuzco: Aguas y Poder, 153 p.; Cuzco: Centro Bartolomé de las Casas.

ZUIDEMA, T., 1990 - Dynastic Structures in Andean Culture. In: The Northern Dynasties. Kingship and Statecraft in Chimor (M. E. Moseley \& A. Cordy-Collins, eds.): 489-506; Washington D.C.: Dumbarton Oaks Research Library and Collection.

ZUIDEMA, T., 2005a - Problèmes de structure dans les Andes. De la parenté, de la polygynie et des moitiés à Cuzco. Journal de la Société des Américanistes, 91(2): 31-49; París.

ZUIDEMA, T., 2005b - La religión inca. In: Religiones Andinas (M. M. Marzal, ed.): 89-113; Madrid: Editorial Trotta.

ZUIDEMA, T., en prensa - El Calendario Inca. Tiempo y Espacio en la Organización Ritual del Cuzco; Lima: Fondo editorial del Congreso, Pontificia Universidad Católica del Perú, Universidad Nacional Mayor de San Marcos.

ZUIDEMA, T. \& POOLE, D. A., 1982 - Los límites de los cuatro suyus incaicos en el Cuzco. Bulletin de I'Institut Français d'Études Andines, 11(1-2): 83-89; Lima.

ZUIDEMA, T. \& URTON, G., 1976 - La Constelación de la Llama en los Andes Peruanos. Allpanchis Phuturinqa, 9: 59-119; Cuzco. 